\title{
Cognitive and Social Influences on Early Prosocial Behavior in Two Sociocultural Contexts
}

\author{
Joscha Kärtner and Heidi Keller \\ University of Osnabrück
}

\author{
Nandita Chaudhary \\ Lady Irwin College, University of Delhi
}

\begin{abstract}
In this cross-cultural study, we tested 2 main hypotheses: first, that an early self-concept along with self-other differentiation is a universal precursor of prosocial behavior in 19-month-olds, and second, that the importance attached to relational socialization goals (SGs) concerning interpersonal responsiveness (obedience, prosocial behavior) is related to toddlers' prosocial behavior. Contrary to these predictions, the results show that mirror self-recognition, as an indicator of early self-concept, was correlated with toddlers' prosociality only in the Berlin sample $(N=38)$ and not in the Delhi sample $(N=39)$. As expected, however, Delhi mothers emphasized relational SGs more strongly than did Berlin mothers. There were no cross-cultural differences in toddlers' prosociality. On an individual level, mothers' emphasis on relational SGs (obedience) was a significant predictor of toddlers' prosocial behavior. On the basis of these results, we propose that situational helping behavior based on shared intentional relations provides an alternative developmental pathway for understanding toddlers' prosocial behavior. This view differs from the often-cited view that anticipating other people as autonomous intentional agents with their own psychological states gives rise to prosocial behavior in toddlers.
\end{abstract}

Keywords: prosocial behavior, empathy, self-other differentiation, culture-specific pathways
Early prosocial behavior emerges in toddlers' second year of life and increases in frequency and variety over this time (ZahnWaxler, Radke-Yarrow, Wagner, \& Chapman, 1992). In response to another person's distress, toddlers begin to show prosocial behavior that is designed to alleviate that person's negative affective state. These behaviors include helping or comforting the distressed person. The consensus in the literature is that early prosocial behavior in these situations is motivated by empathic concern (also sympathy), which can be defined as a vicarious emotional reaction that involves feelings of sorrow or concern for a distressed or needy other (Eisenberg, Fabes, \& Spinrad, 2006; Hoffman, 2000).

Inherent in this definition of empathically motivated prosocial behavior is the notion of self-other differentiation. Hoffman (1975) was the first to theorize that in order to experience empathic concern one must be able to differentiate between what happens to others and what happens to oneself. Subsequent research has followed this line of thought that prosocial behavior is related to the development of an early self-concept and self-other differentiation (Barresi \& Moore, 1996; Bischof-Köhler, 1991; Perner,

Joscha Kärtner and Heidi Keller, Faculty of Human Sciences, Culture, Learning and Development, University of Osnabrück, Osnabrück, Germany; Nandita Chaudhary, Department of Child Development, Lady Irwin College, University of Delhi, New Delhi, India.

This project was funded by German Research Foundation Grant KE263/ 46. Many thanks go to Pooja Bhargava, Astrid Kleis, and Jörn Borke, who helped with assessing and/or coding the data. We are especially grateful to the families that participated in this study.

Correspondence concerning this article should be addressed to Joscha Kärtner, University of Osnabrück, Faculty of Human Sciences, Culture, Learning and Development, 49069 Osnabrück, Germany. E-mail: joscha.kaertner@uni-osnabrueck.de
1991; Zahn-Waxler et al., 1992). The basic idea is that as soon as toddlers develop a sense of themselves and others as separate, independent agents, emotional contagion-an ontogenetically previous response that does not depend on self-other differentiation and results in the observer indiscriminately experiencing the same emotion as the other-may be transformed, at least in part, into a more reciprocal feeling of empathic concern for the distressed other.

Nevertheless, the mechanisms underlying emotional contagion play an important role in motivating prosocial behavior; due to fundamental organizational features of the nervous system (e.g., shared representations for perception and action), the observation of someone in distress induces the same or a very similar emotional reaction in the observer (Preston \& de Waal, 2002). As prefrontal functions increase during the second year, the child is able to identify which feelings are his (or hers) and which feelings belong to the other person (for a review, see Decety \& Jackson, 2004, 2006). Thus, along with advances in inhibitory control and executive functions, the child may experience empathic concern, which then motivates prosocial behavior.

Empirical support for the assumption that empathic concern depends on an early self-concept and self-other differentiation comes from research showing that empathically motivated prosocial behavior correlates with mirror-self-recognition (MSR; Bischof-Köhler, 1989, 1994; Johnson, 1982; Zahn-Waxler et al., 1992). Several lines of research support the interpretation of MSR as an indicator of a more general capacity to represent the self and others as autonomous agents (Moore, 2007; Perner, 1991; Suddendorf \& Whiten, 2001). With regard to self-awareness, there is empirical evidence that MSR correlates with self-conscious emotions such as embarrassment (Lewis, Sullivan, Stanger, \& Weiss, 1989), body self-awareness (Moore, Mealiea, Garon, \& Povinelli, 2007), and pronoun use (Lewis \& Ramsay, 2004). With regard to 
awareness of others, MSR correlates with synchronous imitation (Asendorpf \& Baudonnière, 1993; Asendorpf, Warkentin, \& Baudonnière, 1996) and empathically motivated prosocial behavior (Bischof-Köhler, 1989, 1994; Johnson, 1982; Zahn-Waxler et al., 1992). These correlations were independent of age (with the exception of that reported in Lewis et al., 1989), and the authors reported medium-to-large effect sizes ( $r$ s ranged between .21 and .68). Furthermore, toddlers begin to understand the subjectivity of preferences and interest at around the same age, in the middle of the second year (Repacholi \& Gopnik, 1997; Tomasello \& Haberl, 2003). Given these findings, we hypothesized that, independent of the toddlers' sociocultural background, self-other differentiation should be a universal and necessary social-cognitive precondition of early prosocial behavior. In this way, MSR should be positively correlated with toddlers' prosociality.

It is a central assumption of this study, as in the studies outlined above, that empathically motivated prosocial behavior is based on a biological tendency to react responsively to the distress of others. Toddlers' prosocial behavior should, however, depend on how this tendency is integrated within social interactions and further socialized. Thus, toddlers' natural proclivity to react responsively is expected to abate, stabilize, or increase depending on toddlers' differing socialization experiences. Caretakers generally reinforce or initiate desirable target behavior (for a review, see Eisenberg et al., 2006). For example, Zahn-Waxler, Radke-Yarrow, and King (1979) identified a specific type of parenting behavior that was related to 1- and 2-year-old toddlers' prosocial behavior: Mothers who responded to their toddlers' transgressions with high intensity and clarity, both cognitively and affectively, and who used powerassertive psychological strategies had children who were more likely to help others in situations that they had observed (altruism) or had caused (transgression). Furthermore, 1- and 2-year-olds who were instructed or prompted to help or share tended to do so (Hay \& Murray, 1982), as did toddlers whose parents modeled prosocial behavior (Rheingold, 1982). Other studies have shown that toddlers' participation in prosocial activities also fostered toddlers' prosocial behavior (Keller et al., 2004; Whiting \& Pope Edwards, 1988; Whiting \& Whiting, 1975): In sociocultural contexts such as these, toddlers were involved in routine activities from early on and were expected to help with domestic work and to run errands for their families (Nsamenang, 1992). The results of these studies suggest that children show more prosocial behavior in those contexts in which prosocial behavior is required and encouraged. We would expect that children from cultural contexts in which prosocial behavior and social responsiveness is an important value would show greater prosocial behavior.

It is important to keep in mind that parenting strategies that foster prosocial behavior require a great deal of parental investment. Whether caretakers see the necessity in and are willing to invest effort into socialization should, therefore, depend on their normative background and the importance the caretakers associate with the development of their toddlers' prosocial behavior. Therefore, if norms of interpersonal relatedness (e.g., obedience, sharing, helping) are emphasized within particular cultural contexts, caregivers from these contexts should adopt parenting strategies that require and encourage prosocial behavior from their children. As a consequence, toddlers from these contexts should display a high level of prosocial behavior.
According to cross-cultural perspectives on development, cultural emphases on particular values and socialization toward these values accelerate development in specific domains (Greenfield, Keller, Fuligni, \& Maynard, 2003; Keller, 2007; LeVine \& Norman, 2001; Rothbaum, Pott, Azuma, Miyake, \& Weisz, 2000). In this way, caretakers' socialization goals act as a proximal mechanism by which culture shapes toddlers' prosocial behavior. The consensus in the cross-cultural literature is that empathic concern and prosocial behavior are of central importance in cultural contexts that have been described as interdependent (or collectivistic). In contrast, independent (or individualistic) cultures focus more on individual autonomy (Kağıtçıbaşı, 2007; Keller, 2007; Markus \& Kitayama, 1994; Triandis, 1993). In interdependent sociocultural contexts, values of interpersonal relatedness (e.g., empathic concern, prosocial behavior, obedience) play important roles both as values and guiding principles for an individual's own behavior and as socialization goals for an individual's children (see also Rothbaum et al., 2000).

However, cross-cultural approaches fall short as explanations of the emergence of early prosocial behavior in that they do not take into account the development of self-other differentiation. Although values of interpersonal relatedness may play a role in the emergence of early prosocial behavior, the requisite development of self-other differentiation is rather fostered by a cultural emphasis on autonomy (Keller, Kärtner, Borke, Yovsi, \& Kleis, 2005; Keller et al., 2004). Empathically motivated prosocial behavior is an interesting phenomenon cross-culturally because there are two central intertwining concepts that are often taken to be opposing concepts: autonomy and interpersonal relatedness. In order to help others, toddlers need a sense of autonomy; they must be able to represent themselves and others as autonomous, independent agents. Toddlers also need a sense of interpersonal relatedness in order to help others; the other person's distress must be relevant to the toddlers themselves. Only once these two conditions are met can toddlers feel empathic concern for a needy other or the propensity to alleviate the other's negative state.

Our aim in the present study was to integrate these two strands of research: social-cognitive preconditions and normative influences on early prosocial behavior. Only then would it be possible to evaluate whether toddlers lack the social-cognitive skills or the necessary responsiveness to help others. In this way, we would be able to analyze the concurrence of social-cognitive and motivational influences involved in the genesis of prosocial behavior. We would also be able to identify the specific social or sociocultural influences on the development of prosocial behavior. In order to address these issues, we recruited urban middle-class families from two distinct sociocultural contexts, Berlin, Germany, and Delhi, India.

German middle-class mothers socialize their children toward individuality, autonomy (Keller, 2007), and self-reliance (LeVine $\&$ Norman, 2001). These mothers value exclusive dyadic interaction and believe that children need to spend time on their own in order to become more independent (Keller, Voelker, \& Yovsi, 2005; Keller et al., 2004). In this way, German middle-class families should have a predominantly autonomous sociocultural orientation. Indian middle-class families, on the other hand, strongly emphasize social relationships and interpersonal responsibilities (Chaudhary, 2004; Kumar, 1993; Miller \& Bersoff, 1992; Miller, Bersoff, \& Harwood, 1990; Miller \& Luthar, 1989; Wang 
\& Chaudhary, 2005). In a number of cross-cultural studies, Miller and her colleagues found that Hindu Indians treated interpersonal relations and helping others in fully moral terms (i.e., both as involving a sense of objective obligation and as being within the scope of legitimate regulation). Euro Americans, on the other hand, tended to categorize helping others either as personal-moral issues or as a matter of personal choice (Miller et al., 1990; Miller \& Luthar, 1989). Miller and Bersoff (1992) have also shown that the majority of Hindu Indians from 8 years of age gave priority to interpersonal responsibilities relative to justice considerations, whereas the Euro American participants gave priority to justice considerations relative to interpersonal responsibilities. They argued that this differential emphasis is related to a more intrinsic and obligatory perspective on relationships stressed in Hindu Indian culture as compared to the more voluntaristic view of social relationships stressed in the Euro American culture. At the same time, however, much more emphasis is placed on autonomy by Indian middle-class families than by lower class Indian families because the former are highly educated and their occupations require flexibility and self-determination (Raman, 2003; Sinha \& Tripathi, 1994; Verma \& Saraswathi, 2002). In this way, Indian urban middle-class families should have a predominantly autonomous-relational sociocultural orientation in which autonomy and relatedness hold a similar degree of importance (see Kağıtçıbaşı, 2007).

Given these observations, we hypothesized that Berlin mothers should emphasize autonomous socialization goals more strongly than they should relational socialization goals, whereas Delhi mothers should emphasize autonomy and relatedness to similar degrees. Furthermore, mothers from Delhi should emphasize relational socialization goals more strongly than should mothers from Berlin.

The overarching goal in the present study was to analyze the concurrence of social-cognitive and social influences on prosocial behavior in two different sociocultural contexts. There were three specific hypotheses: First, self-other differentiation should be a universal cognitive precondition of prosocial behavior. In this way, MSR should be positively correlated with the toddlers' prosociality in both samples. Second, a similar number of toddlers should recognize themselves in the mirror in the Berlin and Delhi samples, given that autonomous socialization goals seem to be emphasized to similar degrees in the two cultures. Third, there should be a positive relation between relational socialization goals and the toddlers' prosociality both at the individual and the group level. This means that the more mothers stress relational socialization goals, the more pronounced the toddlers' prosociality should be. Furthermore, the toddlers' prosociality should be more pronounced in the Delhi sample than in the Berlin sample, because prosocial socialization goals should be emphasized more strongly in the Delhi sample.

\section{Method}

\section{Participants}

In Berlin and Delhi, local research assistants recruited families in cooperation with pediatricians and hospitals. There were complete data from 31 of the 38 families from Berlin and 34 of the 39 families from Delhi that participated in the present study. In all other cases toddlers were unwilling to participate in at least one of the tasks $(N=3)$, mothers violated instructions and forced markdirected or prosocial behavior $(N=3)$, or there was a procedural error $(N=6)$.

On average, toddlers in the Berlin sample were 19 months and 4 days $(S D=6.51)$ of age and toddlers in the Delhi sample were 19 months and 8 days $(S D=10.08)$ of age, $t(72)=-2.42, p<$ $.05, d=0.56$. Gender was balanced across the samples (Berlin, $47.2 \%$ female; Delhi, $48.7 \%$ female). The toddlers were the only child (Berlin, 69.4\%; Delhi, 39.5\%), or they had one (Berlin, 19.4\%; Delhi, $60.5 \%$ ) or two (Berlin, 11.1\%; Delhi, 0.0\%) siblings $\left(\chi^{2}=14.99, p<.01\right)$. Mothers from Berlin were significantly older $(M=34.0$ years, $S D=4.12)$ than were mothers from Delhi $(M=29.0$ years, $S D=3.23), t(73)=5.85, p<.01, d=1.35$. Across both samples, mothers had similar educational attainments; the majority of mothers held a high school degree or higher (Berlin, 83.3\%; Delhi, 97.4\%), and, on average, they had received formal education for 15.7 years (Berlin, $M=15.81$ years, $S D=$ 3.35; Delhi, $M=15.62$ years, $S D=1.37$ ). Except for those in two Berlin families, the parents in both samples were living together. The nuclear family was the dominant family type in the Berlin sample $(91.7 \%)$, whereas the extended family was the dominant family type in the Delhi sample (68.4\%). As a consequence, there were more people living in the Delhi households $(M=5.87, S D=$ 2.10) than there were in the Berlin households $(M=3.50, S D=$ $0.78), t(72)=6.52, p<.01, d=1.52$. In all of the extended families in the Delhi sample, there was at least one grandparent living in the same household. Other family members living in the household typically included the parents' siblings (28.9\%).

\section{Procedure and Coding}

Two female experimenters from each cultural context visited the families at home. These home visits lasted about two hours and were part of a larger longitudinal study, in which families were called on when children were 3, 19, 36, and 48 months old. After one of the experimenters had given an overview of the visit and the assessments, the mothers filled in questionnaires regarding sociodemographic information and socialization goals, while the other experimenter established rapport with the toddler. From the behavioral observations and quasi-experimental tasks that followed, we identified two key tasks: the rouge test and a distress simulation. Both assessments were video-recorded by the second experimenter.

Autonomous and relational socialization goals. For assessing maternal socialization goals, we generated items that cover the essential aspects of the two central cultural dimensions (i.e., autonomy and relatedness). Thus, the socialization goals questionnaire consisted of two scales: (a) the Autonomous Socialization Goals Scale, whose items refer to the toddlers' self-confidence and assertiveness (4 items: during the first 3 years of life, children should develop self-confidence; develop assertiveness; develop a sense of self-esteem; develop a sense of self), and (b) the Relational Socialization Goals Scale. The Relational Socialization Goals Scale comprises two subscales: Prosocial Behavior (3 items: learn to help others; care for the well-being of others; cheer up others) and Obedience ( 2 items: learn to obey parents; learn to obey older persons). These two subscales differentiate between what Triandis (2001) described as horizontal collectivism (empa- 
thy and prosocial behavior) and vertical collectivism (respect and obedience; see also Greenfield et al., 2003; Kağıtçıbaş1, 2007; Keller, 2007; Markus \& Kitayama, 1994). Mothers were asked to indicate how important these socialization goals were for them on a 6-point Likert scale.

In order to minimize method and item bias and to ensure that the interpretation of the mean differences between the two cultural samples was appropriate, we tested the questionnaires for structural equivalence. Only if the criteria for structural equivalence are met (i.e., equal factor loadings and, in the case of more than one latent factor, equal variances and covariances of the latent factors across the two samples) can mean differences be interpreted meaningfully (Byrne, 2004). We tested for structural equivalence by using structural equation modeling with AMOS 16.0. Because the sample sizes were small, we did so separately for the two scales, namely, autonomy and relatedness (prosocial behavior and obedience). For the Autonomous Socialization Goals Scale, structural equivalence across cultures was confirmed by a two-group (Berlin and Delhi) structural equation model with cross-cultural equality constraints for factor loadings (measurement weights model). This multigroup model had an adequate fit, $\chi^{2}(7)=9.06, p=.25$, goodness of fit index $=.94$, root mean square error of approximation $=.06$, and model comparisons indicated that this model did not fit worse than the unconstrained model, $\Delta \chi^{2}(3)=0.96$, $p=.92$.

For the Relational Socialization Goals Scale and its two subscales, structural equivalence across cultures was confirmed by a two-group structural equation model with cross-cultural equality constraints for factor loadings, the variances of the two factors, and their covariance (structural covariances model). This model had an adequate fit across cultural groups, $\chi^{2}(15)=11.77, p=.70$, goodness of fit index $=.95$, root mean square error of approximation $<.001$, and model comparisons indicated that this model did not fit worse than either the unconstrained model, $\Delta \chi^{2}(6)=$ $3.42, p=.76$, or the measurement weights model, $\Delta \chi^{2}(3)=0.28$, $p=.96$. Model comparisons further indicated that a one-factorial model (relatedness) represented the data significantly worse than did a two-factorial model (prosocial behavior and obedience), $\Delta \chi^{2}(1)=5.20, p<.05$. Consequently, prosocial behavior and obedience should be treated as distinct, though correlated, factors. Internal consistencies were medium to high for all scales within both samples (Cronbach's alphas ranged between .72 for the Autonomous Socialization Goals Scale in Berlin and .90 for the Obedience Subscale of the Relational Socialization Goals Scale in Delhi).

MSR. The rouge test is the standard procedure for assessing MSR, whereby a red mark is surreptitiously placed on the individual's face before the individual sees him- or herself in a mirror (Amsterdam, 1972; Gallup, 1970). Mirrors were of comparable size in the two sociocultural contexts, and the children could see their whole figure in the mirror. The experimenter explained the procedure and instructed any other people present to keep out of the area reflected in the mirror and to say nothing that might help the toddler localize the mark. The experimenter then set up the covered mirror at a suitable place, leaning it against a wall if possible. In the first phase of the rouge test, the toddler was confronted with his or her mirror image while the researcher and mother sat to either side of the mirror. After approximately three minutes, the mother surreptitiously put a dot of red lipstick on the child's face close to the nose with her index finger while pretending to blow the child's nose or clean the child's face. In the second phase, toddlers were again confronted with their mirror image for five minutes or less if they had displayed clear mark-directed behavior earlier.

Performance on MSR tasks was coded from videotape. Coders evaluated whether or not children showed mark-directed behavior. Mark-directed behavior was defined as the toddlers either touching their own face with an extended index finger while looking in the mirror or pointing toward their own face while turning to and looking at another person. None of the mark-directed behaviors occurred in the first phase of the task. The videos of all participants from both cultural contexts were coded by the first author and one coder from the respective cultural context. There was $95 \%$ agreement for the Berlin sample and $97 \%$ agreement for the Delhi sample (Cohen's $\kappa=.88$ and .94 , respectively). Disagreements $(n=3)$ were resolved by joint recoding.

Prosociality. The toddlers' reactions to distress in others were observed in a quasi-experimental setting, where the experimenter first played with the toddler using a standardized set of toys (e.g., building bricks, puppet, car). Among these toys were two teddy bears, one of which had a hook-and-loop fastener attached to its arm (cf. Bischof-Köhler, 1989, 1991). After 10 min of free play, the experimenter removed the teddy's jacket so that its "fastened" arm broke. She gasped, looked at the teddy, and put it down along with the arm and the jacket and said, "Look! The arm fell off! The arm broke, my teddy is broken!" The experimenter then covered her face and started sobbing. Every 15-20 s, she looked at the teddy and the toddler and repeated the above phrase. After $2.5 \mathrm{~min}$, the experimenter stopped sobbing, told the child that she would take the teddy home to repair it, put it aside, and continued playing with the child for another $5 \mathrm{~min}$ if possible. If the child showed clear prosocial behavior, either by providing physical comfort (e.g., hugging, kissing) or by offering an alternative toy, the experimenter stopped sobbing, thanked the child, and continued playing. The mothers were present during the distress simulation. They sat at a distance of about $3 \mathrm{~m}$ and were instructed to remain silent and to interfere only on direct request of the toddler.

The toddlers' behavior during the distress simulation was coded from videotape. In order to characterize general behavior of the toddlers, we coded four aspects of their behavior: (a) gaze, (b) various distress indicators, (c) exploratory behavior, and (d) prosocial behavior.

Gaze. Coders identified on- and offsets of looking at the experimenter during the distress simulation and the subsequent play interaction (event coding). The final score for the toddlers' gaze was the relative duration of looking at the experimenter, calculated separately for both phases (distress simulation and subsequent play interaction; i.e., percentage of phase duration).

Distress indicators. The distress indicators were (a) freezing (while looking at the experimenter, the child appeared frozen in position and there was no change of gross body position, or the child's body showed signs of tension or rigidity); (b) automanipulation (the child scratched body parts, pulled his or her clothes, or showed atypical body movements or gestures); and (c) stereotypic movements (the child unintentionally manipulated an object, e.g., kept turning a toy around without looking). In order to code these distress indicators, we employed an interval coding approach based on 10-s intervals. For each interval, coders evaluated 
whether the coding criteria applied for at least five seconds, calculated separately for each distress indicator. The final distress score was the relative frequency (i.e., percentage of 10-s intervals) in which at least one of the distress indicators occurred. The score was calculated separately for both phases (distress simulation and play interaction; i.e., percentage of phase duration).

Exploratory behavior. The coders identified two types of behavior during the distress simulation: (a) Toddlers either examined the broken or the intact teddy (e.g., they turned the teddy and the broken arm and had a close look at the position where the arm had been before) or (b) they showed a specific gazing pattern (i.e., they looked first at the sobbing experimenter, then at the broken teddy, and then back at the experimenter). This gazing pattern indicates that toddlers were looking for a possible explanation for the experimenter's distress. The final score for exploratory behavior was the relative frequency of the sum of the two behaviors per minute of the distress simulation.

Prosociality. The principal focus of the analysis of the distress simulation was the toddlers' prosocial behavior. Coders evaluated the toddlers' prosociality on a 3-point scale ranging from 0 to 2 on the basis of toddlers' behavior during the whole episode (distress simulation plus play interaction). Toddlers were awarded a score of 2 (prosocial behavior) if they showed concern for the distressed experimenter (i.e., furrowing or raising of the eyebrows together with a concerned facial expression) and either (a) tried to help her by object-directed prosocial behavior (e.g., trying to repair the teddy, taking the teddy and/or the teddy's arm to the mother) or by person-directed prosocial behavior (e.g., offering an alternative toy, physical comfort) or (b) perseveringly and intensely alarmed their mother (i.e., pointing repeatedly at the experimenter while vocalizing and looking back and forth between the experimenter and the mother). Toddlers were awarded a score of 1 (tentative prosocial behavior) if they seemed concerned and helpless or if they showed ineffective or tentative behavior (e.g., hesitantly alarming their mother or saying words like "ouch," "teddy," or "broken"). Toddlers were awarded a score of 0 (no prosocial behavior) if they were concerned but did not show any prosocial behavior or if they were emotionally unaffected by the experimenter's distress.

Four toddlers from the Berlin sample showed a strong emotional reaction to the experimenter's distress; they started crying uncontrollably and rushed to their mother looking for physical comfort. In these cases, the experimenter stopped the distress simulation immediately and reassured the child that everything was fine. Because these toddlers did not have the opportunity to show helping behavior, they were excluded from further analyses.

Performance on the distress-simulation tasks was coded from videotape by the first author and one coder from each respective cultural context. All coders were blind to the toddlers' performance on the MSR task. In order to compute coding reliabilities, the first author and a second coder from the respective cultural context coded at least $20 \%$ of both samples. Reliabilities were medium to high for gaze $\left(\kappa_{\text {Berlin }}=.81, \kappa_{\text {Delhi }}=.85\right)$, distress $\left(\kappa_{\text {Berlin }}=.78, \kappa_{\text {Delhi }}=.72\right)$, and exploratory behavior $\left(\kappa_{\text {Berlin }}=\right.$ $\left..71, \kappa_{\text {Delhi }}=.75\right)$. All of the videos were coded with respect to prosocial behavior by two coders, and the reliabilities were medium to high $\left(\kappa_{\text {Berlin }}=.84, \kappa_{\text {Delhi }}=.80\right)$. Where there were disagreements between the coders, the coders discussed each disagreement and reached an agreement for all but one case in each cultural context. These two cases were excluded from further analyses.

\section{Results}

In the first part of this section we present the data regarding cross-cultural similarities and differences in autonomous and relational socialization goals and descriptive information on MSR and the toddlers' behavior during the distress simulation. In the second part of this section, we test the hypotheses concerning cognitive preconditions and normative influences on the toddlers' prosocial behavior separately for the two cultural groups as well as for the sample as a whole.

\section{Autonomous and Relational Socialization Goals}

In order to determine whether the two cultural groups differed in terms of their socialization goals (SGs), we subjected the data to a repeated-measures analysis of variance (ANOVA) with the SG (autonomous, prosocial, and obedience) as the within-subjects factor and cultural group (Berlin or Delhi) as the between-subjects factor. This analysis yielded a significant main effect of SG, $F(2$, $150)=7.19, p<.01, \eta^{2}=.09$; collapsing across cultural group, autonomous SGs were emphasized significantly more $(M=4.47$, $S D=1.17)$ than were prosocial SGs $(M=4.03, S D=1.11)$, $t(76)=-2.91, p<.01$, and obedience SGs, $(M=4.12, S D=$ $1.25), t(76)=-1.78, p<.10$. There was also a significant $\mathrm{SG} \times$ Cultural Group interaction, $F(2,150)=48.25, p<.001, \eta^{2}=.39$. Inspection of the means (see Table 1) indicates that the relative emphases on autonomous and relational (prosocial and obedience) SGs differed between the two cultural groups: Berlin mothers placed greater emphasis on autonomous SGs and emphasized relational SGs to a lesser degree than did Delhi mothers. This effect held for both of the relational subscales (prosocial and obedience SGs) but was more pronounced for obedience (see Table 1).

\section{MSR}

As expected, the proportion of toddlers who recognized themselves in the mirror was similar in Berlin and Delhi. About $70 \%$ of toddlers in each cultural group recognized themselves in the mirror (see Table 1).

\section{Toddlers' Behavior During the Distress Simulation}

All except two toddlers (97\%) looked longer at the experimenter during the distress simulation than during the play interaction. The same number of toddlers showed distress during the distress simulation for at least one 10-s interval. The percentage of toddlers who showed distress during the subsequent play interaction for at least one 10 -s interval dropped to $50 \%$. In order to examine any differences in toddlers' gaze and distress between the distress simulation and the play interaction, we computed paired $t$ tests for the total sample given that there was no difference between the two cultural groups for either variable (see Table 1). As expected, the toddlers gazed much longer at the experimenter during the distress simulation $(M=0.41, S D=0.16)$ than they did during the play interaction $(M=0.14, S D=0.13), t(65)=13.32, p<.01, \eta^{2}=$ .73. The toddlers were also distressed for longer during the distress 
Table 1

Cross-Cultural Differences in Socialization Goals (SGs), Mirror Self-Recognition (MSR), and Prosocial Behavior

\begin{tabular}{lccc}
\hline \multicolumn{1}{c}{ Variable } & Berlin & Delhi & Statistics \\
\hline Autonomous SGs & $5.09(0.80)$ & $3.87(1.16)$ & $t(75)=5.35^{* * * *}, d=1.23$ \\
Relational SGs & $3.82(1.16)$ & $4.24(1.03)$ & $t(75)=-1.70^{\dagger}, d=0.39$ \\
$\quad$ Prosocial & $3.50(1.13)$ & $4.73(1.06)$ & $t(75)=-4.95^{* * * *}, d=1.13$ \\
$\quad$ Obedience & $69.4 \%$ & $70.3 \%$ & $\chi^{2}=0.01$ \\
$\quad$ MSR & $0.43(0.17)$ & $0.39(0.16)$ & $t(64)=1.03, d=0.23$ \\
Distress simulation & $0.48(0.21)$ & $0.46(0.23)$ & $t(64)=0.44, d=0.10$ \\
$\quad$ Gaze (relative duration) & $1.13(0.90)$ & $1.18(0.95)$ & $t(64)=-0.21, d=-0.05$ \\
$\quad$ Distress (relative duration) & $1.04(0.73)$ & $0.92(0.89)$ & $t(64)=0.56, d=0.13$ \\
$\quad$ Exploratory behavior (relative frequency) & & &
\end{tabular}

Note. Parenthetical values are standard deviations. For MSR, $N_{\text {Berlin }}=36, N_{\text {Delhi }}=37$. For distress simulation, $N_{\text {Berlin }}=29, N_{\text {Delhi }}=37$.

${ }^{\dagger} p<.05$, one-tailed. ${ }^{* * * *} p<.001$, two-tailed.

simulation $(M=0.47, S D=0.22)$ than they were during the play interaction $(M=0.13, S D=0.22), t(65)=10.91, p<.01, \eta^{2}=$ .65 .

Most toddlers showed exploratory behavior; $85 \%$ of toddlers showed at least one exploratory behavior, and, on average, toddlers showed $1.16(S D=0.92)$ such behaviors per minute. This figure did not differ between the two cultural groups, $t(64)=-0.21, p>$ .10 (see Table 1).

With regard to prosocial behavior, some of the toddlers intervened prosocially when faced with the experimenter's distress. In Berlin, $27.6 \%$ of the toddlers showed prosocial behavior and $48.3 \%$ of the toddlers showed tentative prosocial behavior. In Delhi, $35.1 \%$ of the toddlers showed prosocial behavior and $21.6 \%$ of the toddlers showed tentative prosocial behavior. These distributions differed between cultural groups on a marginally significant level $\left(\chi^{2}=5.46, p<.10\right)$. The mean scores of the toddlers' prosocial behavior did not differ between the Berlin and Delhi samples, $t(64)=0.56, p>$.10. Including the four toddlers who showed a strong emotional reaction as either tentatively prosocial (1) or not prosocial (0) did not change the results.

\section{Social-Cognitive and Normative Influences on Prosocial Behavior}

The data regarding social-cognitive and social influences on prosocial behavior were first subjected to simple correlation analyses and then to a hierarchical regression analysis. Because we hypothesized that self-other differentiation should be a cognitive precondition of prosocial behavior regardless of the sociocultural context, MSR should be correlated with the toddlers' prosocial behavior in both samples. The point-biserial correlation was significant only for the Berlin sample $\left(r=.37, p_{\text {one-tailed }}<.05\right)$ and not for the Delhi sample $\left(r=-.19, p_{\text {one-tailed }}>.10\right)$. These two correlation coefficients differed significantly from each other $(z=$ $2.14, p<.05)$.

We also hypothesized that there should be a positive relation between relational SGs (prosocial behavior and obedience) and the toddlers' prosocial behavior both at the individual and the group level. There were consistent positive correlations between SGs and prosocial behavior, but only the obedience SGs were significantly correlated with prosocial behavior in both samples (Berlin, $r=$ $.30, p_{\text {one-tailed }}<.10$; Delhi, $\left.r=.41, p_{\text {one-tailed }}<.01\right)$. Table 2

Table 2

Correlations Between Socialization Goals (SGs), Mirror Self-Recognition (MSR), and Distress Simulation for the Berlin and Delhi Samples

\begin{tabular}{|c|c|c|c|c|c|c|c|c|}
\hline Variable & 1 & 2 & 3 & 4 & 5 & 6 & 7 & 8 \\
\hline 1. Autonomous SGs & - & $.55^{* *}$ & $.48^{* *}$ & .07 & .04 & -.27 & .11 & .21 \\
\hline 2. Prosocial SGs & $.41^{*}$ & - & $.58^{* *}$ & .00 & .05 & -.04 & .04 & .21 \\
\hline 3. Obedience & .25 & $.59^{* *}$ & - & -.02 & .27 & .10 & .13 & $.41^{\dagger \dagger}$ \\
\hline 4. MSR & -.05 & .12 & -.02 & - & .22 & -.01 & -.19 & -.19 \\
\hline 5. Gaze & -.06 & -.03 & -.14 & -.20 & - & $.57^{* * * *}$ & -.16 & -.10 \\
\hline 6. Distress & .29 & -.14 & -.09 & -.02 & .22 & - & -.23 & $-.50^{* *}$ \\
\hline 7. Exploration & -.05 & .14 & .09 & .18 & .06 & .10 & - & .23 \\
\hline 8. Prosocial behavior & .13 & .10 & $.30^{(\dagger)}$ & $.37^{\dagger}$ & -.04 & .11 & .17 & - \\
\hline
\end{tabular}

Note. Data below the diagonal represent correlations for the Berlin sample $(N=27)$, and data above the diagonal represent correlations for the Delhi sample $(N=34)$. Note that the pattern of significant results for each sample is identical if one applies pairwise deletion $($ Berlin, $N \leq 38 ;$ Delhi, $N \leq 39)$ instead of listwise deletion of missing cases.

${ }^{\dagger} p<<.10$, one-tailed. ${ }^{\dagger} p<.05$, one-tailed. ${ }^{\dagger \dagger} p<.01$, one-tailed. ${ }^{*} p<.05$, two-tailed. ${ }^{* * *} p<.01$, two-tailed. ${ }^{* * * *} p<.001$, two-tailed. 
shows a full correlation matrix of all measures (i.e., mothers' SGs and toddlers' MSR, gaze, distress, explorative, and prosocial behavior). The three SG subscales were positively intercorrelated within each sample (see Table 2). For the Delhi sample only, there were significant correlations between gaze and distress $(r=.57$, $p<.001)$ and between distress and prosocial behavior $(r=-.50$, $p<.01)$ during the distress episode. For the Berlin sample only, there was a significant correlation between MSR and prosocial behavior. Thus, the only consistent correlation between both samples was between obedience SGs and prosocial behavior.

Simple correlations do not take into account intercorrelations with other predictors of prosocial behavior or any potential confounds, so we computed a hierarchical regression analysis. Toddler age and gender were entered in the first step; cultural group was entered in the second step; and MSR, prosocial SGs, and obedience were entered in the third step. The interaction term Cultural Group $\times$ MSR was entered in the fourth step to account for the culture-specific correlations documented above. Because interaction effects are generally underestimated and moderation effects are difficult to detect, we have adopted Aiken and West's (1991) and Pedhazur's (1997) interpretation method of using interactionterm coefficients with a $p$ value of .10 or less to indicate significance.

We screened for multicollinearity by looking at the tolerances $\left(1-R^{2}\right)$ and conditioning indexes, as suggested by Tabachnik \& Fidell (2007): Tolerances were good: .59 for prosocial SGs and .50 for obedience. In addition, none of the conditioning indexes were greater than $30\left(\mathrm{CI}_{\max }=15.8\right.$ for the interaction term).

As shown in Table 3, the marginally significant betas for gender and age (Step 1) indicate that (a) boys were more prosocial than were girls and (b) younger toddlers were more prosocial than were older toddlers. Cultural group (Step 2) did not affect toddlers' prosocial behavior. When MSR, prosocial SGs, and obedience were entered in Step 3, the proportion of variance explained by the factors entered in Steps 1 and 2 increased significantly from $R^{2}=$ .10 to $R^{2}=.23$. Obedience $(\beta=.36, p<.05)$ was the only significant predictor of prosocial behavior out of all of the additional variables entered at Step 3. The interaction term Cultural Group $\times$ MSR $(\beta=.48, p<.10)$ that was entered in Step 4 significantly increased the proportion of variance explained to $R^{2}=.28$.

\section{Discussion}

Our objective in the present study was to delineate the effects of social-cognitive and other social factors on the emergence of prosocial behavior in 19-month-old toddlers from two different sociocultural settings. Samples from Berlin and Delhi were selected because of their distinctly different sociocultural orientations. The results show that the Berlin mothers emphasized autonomous socialization goals more strongly and relational socialization goals (prosociality and obedience) to a lesser degree than did Delhi mothers, clearly supporting the hypothesized differences in normative orientations. We hypothesized, on the basis of earlier research (Bischof-Köhler, 1989, 1994; Johnson, 1982), that there would be a universal relation between prosocial behavior and MSR. We used MSR as an indicator of the capacity of toddlers to represent themselves and others as autonomous intentional agents. Contrary to our predictions, however, the cross-cultural difference in the mothers' normative orientations turned out to be related to the development of early prosocial behavior in a different way. In particular, MSR and the toddlers' prosocial behavior were positively correlated only in the Berlin sample. There was no significant correlation between MSR and prosocial behavior in the Delhi sample. The question arises, therefore, of whether self-other differentiation is a necessary precondition of all early prosocial behavior.

\section{Situational Helping Behavior}

One could argue, on the basis of theoretical approaches that explain how toddlers may understand the intentionality of an action without attributing mental states to self or to another (e.g., Barresi \& Moore, 1996; Gergely, 2002; Moore, 2007; Reddy, 2008), that there may be an alternative mechanism underlying early helping behavior that is based on emotional contagion instead of empathic concern. According to Barresi and Moore (1996), toddlers come to understand mental states by integrating first-person information (i.e., inner experience) with third-person information (i.e., the agent's observable behavior). Barresi and Moore proposed that there is a developmental stage that precedes toddlers' ability to habitually complement others' behavior by ascribing subjective experience to the other person. This developmental stage is referred to as shared intentional relations: Toddlers come to understand mental states by matching the intentional activities of the self and other while engaging in the same objectdirected activity. In situations where a toddler engages in objectdirected imitation or triadic interaction, therefore, he or she has direct access to information concerning the inner experience of the same psychological or mental state that underlies the observable behavior in the other person. As a consequence, the toddler's

Table 3

Hierarchical Regression of Toddlers' Prosocial Behavior

\begin{tabular}{lcccccc}
\hline \multicolumn{1}{c}{ Predictor } & $\beta_{\text {Step } 1}$ & $\beta_{\text {Step } 2}$ & $\beta_{\text {Step3 }}$ & Final $\beta$ & $R^{2}$ & $\Delta R^{2}$ \\
\hline Gender & $-.30^{*}$ & $-.30^{*}$ & $-.30^{*}$ & $-.24^{(*)}$ & $.10^{*}$ & $.10^{*}$ \\
Age & $-.22^{(*)}$ & -.21 & $-.25^{(*)}$ & $-.23^{* *}$ & & \\
Cultural group & & .05 & .19 & -.15 & $.11^{(*)}$ & .00 \\
MSR & & & .12 & -.08 & $.23^{*}$ & $.13^{*}$ \\
Prosocial SGs & & .02 & -.02 & & \\
Obedience & & $.36^{*}$ & $.40^{*}$ & & $.48^{*}$ \\
Cultural Group $\times$ MSR & & & & $.28^{*}$ & $.05^{(*)}$ \\
\hline
\end{tabular}

Note. $\quad N_{\text {Berlin }}=29, N_{\text {Delhi }}=37$. MSR $=$ mirror self-recognition; SGs $=$ socialization goals

${ }^{(*)} p<.10 . * p<.05$. 
understanding is situation specific and spans the self and other. Barresi and Moore's basic argument is that toddlers understand intentional actions by experiencing and sharing the same psychological or mental state as the other person. Hence, toddlers understand the intentionality of an observed action without ascribing their own or independent experience to the other person.

This principle can similarly be applied to early prosocial behavior in emotionally charged situations: If toddlers observe the emotional expression of another person, this induces-via emotional contagion - a similar emotion in them. As a result, toddlers experience the situation as sad or painful while the other person's object-directed behavior indicates a possible reason for the distress. One could argue that during the distress-simulation phase of this study, the toddlers acquired a situation-specific understanding ("sad because of the broken teddy") although "sad" was not understood as the mental state of the other person. This experience-bound understanding allows toddlers to help the distressed other. Thus, we propose that situational helping behavior is an alternative to empathically motivated helping behavior in emotion-laden situations with a needy or distressed other.

As a consequence of the shared intentional relations principle, self-other differentiation would be, by definition, a precondition of empathically motivated helping behavior in this situation, but it would not be a precondition for helping behavior in all emotionally charged situations, per se. Thus, in our view, situational helping behavior is not a developmentally earlier form of prosocial behavior; rather, it is an equal alternative. In other words, toddlers may develop the competence to act prosocially via different developmental pathways (i.e., empathically motivated vs. situational helping behavior). Common to both pathways is that prosocial behavior (a) is initiated by emotional contagion while observing another person in distress and (b) requires some form of responsiveness that motivates prosocial behavior.

\section{Culture-Specific Developmental Pathways}

We propose, on the basis of Keller's (2007) model of culturally informed developmental pathways, that, depending on the sociocultural context, toddlers may follow different pathways to the same developmental outcome, in this case, early prosocial behavior. The significant correlation between MSR and prosocial behavior for the Berlin toddlers supports the interpretation that helping behavior is predominantly empathically motivated. The finding that MSR was not related to prosocial behavior for the Delhi toddlers, on the other hand, suggests that both empathically motivated and situational helping behavior were evident in this sample.

The idea of culture-specific pathways raises the question of why empathically motivated and situational helping behavior occur to different degrees in different sociocultural contexts. We argue that one of the core features of situational helping behavior is that it does not rely on the toddlers' sense of themselves as autonomous and independent intentional agents. Situational helping behavior should, therefore, be more prominent in sociocultural contexts in which caretakers focus less on their toddlers' mental states, subjective experiences, and inner states than in contexts that are prototypically autonomous (e.g., Western urban middle class; Bischof-Köhler, 1989, 1994; Johnson, 1982). In more relatednessoriented contexts, toddlers may show similar situational helping behavior by joining in the observed situation and reaching a situation-specific understanding. The socialization goal patterns of the Berlin and Delhi mothers support this interpretation.

From the evidence discussed above, it seems unlikely that helping behavior depends on self-other differentiation as a universally necessary social-cognitive precondition. We propose two different possibilities for why helping behavior in emotion-laden situations nevertheless emerges only toward the latter half of the second year: First, it could be that younger toddlers lack the motivation to help others or, more generally, the basic and responsive orientation toward others. Second, helping in these situations presupposes advanced emotion regulation capacities (Eisenberg \& Fabes, 2006). Only if the toddler has the ability to regulate negative affect can the toddler focus his or her attention on the situation at hand and act constructively.

\section{What Is the True Nature of Early Prosocial Behavior?}

Of the two subscales of relational socialization goals it was obedience, and not prosociality, that consistently predicted the toddlers' prosocial behavior in the distress simulation. This finding is consistent with Whiting and Whiting's (1975) and de Guzman, Edwards, and Carlo's (2005) findings that the early assignment of responsibility (e.g., family chores and sibling caretaking) best explained cross-cultural differences in children's prosocial behavior. Graves and Graves (1983) came to a similar conclusion based on their fieldwork in the Cook Islands, Polynesia. They found that girls showed prosocial behavior significantly more often than did boys. From these findings they suggested that there are genderspecific pathways to helping behavior; while girls learned prosocial behavior by being included in meaningful cooperative household work with elders and nurturing younger, more dependent family members, boys learned prosocial behavior in the context of egalitarian peer play.

These findings raise questions concerning the nature of the motivation underlying early helping behavior. If we were to ask whether 19-month-old toddlers help others because they are inherently altruistic or because their sociocultural environment shapes them to be that way, we would say that the answer is both. We propose that toddlers have an evolved, natural proclivity to act prosocially and that socialization practices build on these tendencies, working in concert rather than in conflict with the toddlers' natural predispositions. The present findings suggest that caretakers' emphasis on obedience as a socialization goal influences toddlers' responsive orientation toward others. Thus, it seems that caretakers who stress obedience as a socialization goal use certain socialization practices (e.g., instructing, prompting, modeling, requesting specific behavior) that are effective in shaping their toddlers' predisposition to act prosocially.

Another qualitatively different mechanism that has been described as effective in strengthening toddlers' prosocial behavior is guilt. As Zahn-Waxler et al. (1979) and Hoffman (2000) suggest, the motive for helping others might emanate from some early form of guilt felt for the other's distress, that is, empathy-based (transgression) guilt. In Zahn-Waxler et al.'s study, mothers whose communications toward toddlers' transgressions were of high intensity and clarity had children who were more likely to help others, even in situations that they had only observed. In line with this view, it would be worthwhile for future research to analyze 
how mothers' explicit socialization goals concerning toddlers' development of autonomy and relatedness translate into parenting strategies designed to foster prosocial development of their toddlers. These types of analyses would allow us to pinpoint the nature of the motives underlying early prosocial behavior.

The present study has a number of strengths in that it provides new evidence for normative influences on, and culture-specific pathways to, early prosocial behavior. There are some limitations, however. First, the observations came from contexts that represent autonomous and autonomous-relational sociocultural orientations: highly educated middle-class families in Berlin and Delhi. One has to be cautious about generalizing these data beyond these specific sociocultural contexts. These results need to be replicated by research within other sociocultural contexts that emphasize the same values to determine whether generalizing these data to similar sociocultural contexts in the same or in other countries is appropriate. Second, from looking at the toddlers' prosocial behavior alone, we could not identify behavioral criteria that reliably differentiated between empathically motivated and situational helping behavior. For instance, one might speculate that, in contrast to situational helping behavior, empathically motivated helping behavior is more other-regarding, which could lead to more comforting or less teddy-directed behavior. However, specific behaviors were ambiguous (e.g., alarming) or had too low frequencies (e.g., comforting) for us to conduct these analyses. Thus, tasks that make this differentiation possible should be developed in future research. Third, in the present study, a high proportion of toddlers recognized themselves in the mirror in both samples. It may be beneficial to perform the tests at an earlier age in order to have an equal distribution of recognizers and nonrecognizers.

To sum up, we analyzed the concurrence of social-cognitive and social influences on the development of early prosocial behavior in two distinct sociocultural contexts in the present study. Relational socialization goals, especially obedience, were consistent predictors of toddlers' prosocial behavior. Furthermore, these data indicate that there may be culture-specific developmental pathways to prosocial behavior. In sociocultural contexts that favor autonomy over relatedness, toddlers' prosocial behavior seems to be grounded in empathically perceiving the subjective state that the other person is in. In sociocultural contexts that stress relational aspects of development more strongly, on the other hand, prosocial behavior may have its origins in the joint experience of the other person's distress. We propose that situational helping behavior may be an alternative mechanism for prosocial behavior that does not rely on the perception of others as autonomous agents.

\section{References}

Aiken, L. S., \& West, S. G. (1991). Multiple regression: Testing and interpreting interactions. Newbury Park, CA: Sage.

Amsterdam, B. (1972). Mirror self-image reactions before age two. Developmental Psychobiology, 5, 297-305.

Asendorpf, J. B., \& Baudonnière, P. M. (1993). Self-awareness and otherawareness: Mirror self-recognition and synchronic imitation among unfamiliar peers. Developmental Psychology, 29, 88-95.

Asendorpf, J. B., Warkentin, V., \& Baudonnière, P. M. (1996). Selfawareness and other-awareness II: Mirror self-recognition, social contingency awareness, and synchronic imitation. Developmental Psychology, 32, 313-321.
Barresi, J., \& Moore, C. (1996). Intentional relations and social understanding. Behavioral and Brain Sciences, 19, 107-154.

Bischof-Köhler, D. (1989). Spiegelbild und Empathie. Die Anfänge der sozialen Kognition [Mirror image and empathy: The beginnings of social cognition]. Bern, Switzerland: Huber.

Bischof-Köhler, D. (1991). The development of empathy in infants. In M. E. Lamb \& H. Keller (Eds.), Infant development: Perspectives from German-speaking countries (pp. 245-273). Hillsdale, NJ: Erlbaum.

Bischof-Köhler, D. (1994). Selbstobjektivierung und fremdbezogene Emotionen. Identifikation des eigenen Spiegelbildes, Empathie und prosoziales Verhalten im 2. Lebensjahr [Self-objectification and otheroriented emotions: Self-recognition, empathy, and prosocial behavior in the second year]. Zeitschrift für Psychologie, 202, 349-377.

Byrne, B. M. (2004). Testing for multigroup invariance using AMOS Graphics: A road less traveled. Structural Equation Modeling, 11, 272300.

Chaudhary, N. (2004). Listening to culture: Constructing reality from everyday talk. London, England: Sage.

Decety, J., \& Jackson, P. L. (2004). The functional architecture of human empathy. Behavioral and Cognitive Neuroscience Reviews, 3, 406-412.

Decety, J., \& Jackson, P. L. (2006). A social-neuroscience perspective on empathy. Current Directions in Psychological Science, 15, 54-58.

de Guzman, M. R. T., Edwards, C. P., \& Carlo, G. (2005). Prosocial behaviors in context: A study of the Gikuyu children of Ngecha, Kenya. Journal of Applied Developmental Psychology, 26, 542-558.

Eisenberg, N., \& Fabes, R. A. (2006). Emotion-related regulation and children's socioemotional competence. In L. Balter \& C. S. TamisLeMonda (Eds.), Child psychology: A handbook of contemporary issues (2nd ed., pp. 357-381). New York, NY: Psychology Press.

Eisenberg, N., Fabes, R. A., \& Spinrad, T. L. (2006). Prosocial development. In N. Eisenberg, W. Damon, \& R. M. Lerner (Eds.), Handbook of child psychology: Vol. 3. Social, emotional, and personality development (6th ed., pp. 646-718). New York, NY: Wiley.

Gallup, G. G. (1970, January 2). Chimpanzees: Self-recognition. Science, $167,86-87$

Gergely, G. (2002). The development of understanding self and agency. In U. Goswami (Ed.), The Blackwell handbook of childhood cognitive development (pp. 26-46). Oxford, England: Blackwell.

Graves, N. B., \& Graves, T. D. (1983). The cultural context of prosocial development: An ecological model. In D. L. Bridgeman (Ed.), The nature of prosocial development: Interdisciplinary theories and strategies (pp. 243-264). New York, NY: Academic Press.

Greenfield, P. M., Keller, H., Fuligni, A., \& Maynard, A. (2003). Cultural pathways through universal development. Annual Review of Psychology, 54, 461-490.

Hay, D. F., \& Murray, P. (1982). Giving and requesting: Social facilitation of infants' offers to adults. Infant Behavior and Development, 5, 301310 .

Hoffman, M. L. (1975). Developmental synthesis of affect and cognition and its implications for altruistic motivation. Developmental Psychology, 11, 607-622.

Hoffman, M. L. (2000). Empathy and moral development: Implications for caring and justice. Cambridge, England: Cambridge University Press.

Johnson, D. B. (1982). Altruistic behavior and the development of the self in infants. Merrill-Palmer Quarterly, 28, 379-388.

Kağıtçıbaşı, Ç. (2007). Family, self, and human development across cultures: Theory and applications (2nd ed.). Mahwah, NJ: Erlbaum.

Keller, H. (2007). Cultures of infancy. Mahwah, NJ: Erlbaum.

Keller, H., Kärtner, J., Borke, J., Yovsi, R., \& Kleis, A. (2005). Parenting styles and the development of the categorical self: A longitudinal study on mirror self-recognition in Cameroonian Nso and German families. International Journal of Behavioral Development, 29, 496-504.

Keller, H., Voelker, S., \& Yovsi, R. (2005). Conceptions of parenting in 
different cultural communities: The case of West African Nso and Northern German women. Social Development, 14, 158-180.

Keller, H., Yovsi, R., Borke, J., Kärtner, J., Jensen, H., \& Papaligoura, Z. (2004). Developmental consequences of early parenting experiences: Self-recognition and self-regulation in three cultural communities. Child Development, 75, 1745-1760.

Kumar, K. (1993). Study of childhood and family. In T. S. Saraswathi \& B. Kaur (Eds.), Human development and family studies in India: An agenda for research and policy (pp. 67-76). New Delhi, India: Sage.

LeVine, R. A., \& Norman, K. (2001). The infant's acquisition of culture: Early attachment re-examined in anthropological perspective. In C. C. Moore \& H. F. Mathews (Eds.), The psychology of cultural experience (pp. 83-104). Cambridge, England: Cambridge University Press.

Lewis, M., \& Ramsay, D. (2004). Development of self-recognition, personal pronoun use, and pretend play during the 2nd year. Child Development, 75, 1821-1831.

Lewis, M., Sullivan, M. W., Stanger, C., \& Weiss, M. (1989). Self development and self-conscious emotions. Child Development, 60, 146156 .

Markus, H. R., \& Kitayama, S. (1994). The cultural construction of self and emotion: Implications for social behavior. In S. Kitayama \& H. R. Markus (Eds.), Emotion and culture: Empirical studies of mutual influence (pp. 89-130). Washington, DC: American Psychological Association.

Miller, J. G., \& Bersoff, D. M. (1992). Culture and moral judgment: How are conflicts between justice and interpersonal responsibilities resolved? Journal of Personality and Social Psychology, 62, 541-554.

Miller, J. G., Bersoff, D. M., \& Harwood, R. L. (1990). Perceptions of social responsibilities in India and in the United States: Moral imperatives or personal decisions? Journal of Personality and Social Psychology, 58, 33-47.

Miller, J. G., \& Luthar, S. (1989). Issues of interpersonal responsibility and accountability: A comparison of Indians' and Americans' moral judgments. Social Cognition, 7, 237-261.

Moore, C. (2007). Understanding self and other in the second year. In C. A. Brownell \& C. B. Kopp (Eds.), Socioemotional development in the toddler years: Transitions and transformations (pp. 43-65). New York, NY: Guilford Press.

Moore, C., Mealiea, J., Garon, N., \& Povinelli, D. J. (2007). The development of body self-awareness. Infancy, 11, 157-174.

Nsamenang, B. A. (1992). Perceptions of parenting among the Nso of Cameroon. In B. S. Hewlett (Ed), Father-child relations: Cultural and biosocial contexts (pp. 321-343). Hawthorne, NY: de Gruyter.

Pedhazur, E. J. (1997). Multiple regression in behavioral research. Fort Worth, TX: Harcourt Brace College.

Perner, J. (1991). Understanding the representational mind. Cambridge, MA: MIT Press.

Preston, S. D., \& de Waal, F. B. M. (2002). Empathy: Its ultimate and proximate bases. Behavioral and Brain Sciences, 25, 1-20.

Raman, V. (2003). The diverse life-worlds of Indian childhood. In M. Pernau, I. Ahmad, \& H. Reifeld (Eds.), Family and gender: Changing values in Germany and India (pp. 84-111). New Delhi, India: Sage.
Reddy, V. (2008). How infants know minds. Cambridge, MA: Harvard University Press.

Repacholi, B. M., \& Gopnik, A. (1997). Early reasoning about desires: Evidence from 14- and 18-month-olds. Developmental Psychology, 33, $12-21$.

Rheingold, H. L. (1982). Little children's participation in the work of adults, a nascent prosocial behavior. Child Development, 53, 114-125.

Rothbaum, F., Pott, M., Azuma, H., Miyake, K., \& Weisz, J. (2000). The development of close relationships in Japan and the United States: Paths of symbiotic harmony and generative tension. Child Development, 71, 1121-1142.

Sinha, D., \& Tripathi, R. C. (1994). Individualism in a collective culture: A case of coexistence of opposites. In U. Kim, H. C. Triandis, Ç. Kağıtçıbaşı, S. Choi, \& G. Yoon (Eds.), Individualism and collectivism: Theory, method and applications (pp. 123-136). Thousand Oaks, CA: Sage.

Suddendorf, T., \& Whiten, A. (2001). Mental evolution and development: Evidence for secondary representation in children, great apes, and other animals. Psychological Bulletin, 127, 629-650.

Tabachnik, B., \& Fidell, L. (2007). Using multivariate statistics (5th ed.) Boston, MA: Pearson.

Tomasello, M., \& Haberl, K. (2003). Understanding attention: 12- and 18-month-olds know what is new for other persons. Developmental Psychology, 39, 906-912.

Triandis, H. C. (1993). Collectivism and individualism as cultural syndromes. Cross-Cultural Research, 27, 155-180.

Triandis, H. C. (2001). Individualism-collectivism and personality. Journal of Personality, 69, 907-924.

Verma, S., \& Saraswathi, T. S. (2002). Adolescence in India: Street children or Silicon Valley millionaires. In B. B. Brown, R. W. Larson, \& T. S. Saraswathi (Eds.), The world's youth: Adolescence in eight regions of the globe (pp. 105-140). Cambridge, England: Cambridge University Press.

Wang, Q., \& Chaudhary, N. (2005). The self. In K. Pawlik \& G. d'Ydewalle (Eds.), Psychological concepts: An international historical perspective (pp. 325-358). Hove, England: Psychology Press.

Whiting, B. B., \& Pope Edwards, C. (1988). Children of different worlds: The formation of social behavior. Cambridge, MA: Harvard University Press.

Whiting, B. B., \& Whiting, J. W. (1975). Children of six cultures: A psycho-cultural analysis. Cambridge, MA: Harvard University Press.

Zahn-Waxler, C., Radke-Yarrow, M., \& King, R. A. (1979). Child rearing and children's prosocial initiations toward victims of distress. Child Development, 50, 319-330.

Zahn-Waxler, C., Radke-Yarrow, M., Wagner, E., \& Chapman, M. (1992). Development of concern for others. Developmental Psychology, 28, 126-136.

Received January 16, 2009

Revision received March 9, 2010 Accepted April 2, 2010 